\title{
Primary cardiac involvement of anti-phospholipid syndrome misdiagnosed as infective endocarditis
}

Fatma Nihan Turhan Caglar ${ }^{1}$, Ismail Biyik², Nilgun Isiksacan², Ibrahim Faruk Aktürk ${ }^{1}$

${ }^{1}$ Department of Cardiology, Bakirkoy Dr. Sadi Konuk Education and Research Hospital, Istanbul, Turkey

2Department of Cardiology, Usak University Education and Research Hospital, Usak, Turkey

${ }^{3}$ Department of Biochemistry, Bakirkoy Dr. Sadi Konuk Education and Research

Hospital, Istanbul, Turkey

Submitted: 11 March 2017

Accepted: 12 June 2017

Arch Med Sci Civil Dis 2017; 2: e89-e91

DOI: https://doi.org/10.5114/amscd.2017.68653

Copyright @ 2017 Termedia \& Banach

Anti-phospholipid syndrome (APS) is defined by thrombosis (venous, arterial, or small vessel) and/or pregnancy morbidity (recurrent miscarriage, fetal loss, or placental insufficiency) occurring in the presence of persistently positive anti-phospholipid antibodies (aPL), lupus anticoagulant (LAC) test, anti-cardiolipin antibodies (aCL), and anti-B2-glycoprotein I antibodies [1]. Anti-phospholipid syndrome can occur as an isolated condition or can be associated with connective tissue diseases, most commonly systemic lupus erythematosus (SLE). Herein we present a patient with aPL syndrome and cardiac involvement, misdiagnosed and treated as infective endocarditis.

A 31-year-old woman was admitted to the neurology clinic because of weakness of the left hand. Her clinical history revealed mild weakness of bilateral hands lasting less than $2 \mathrm{~h}$ for about 5 years. Cranial contrast magnetic resonance imaging (MRI) showed atrophic gliotic changes and thin strip style gyral hyperintensity in T1 imaging at the superior sylvian fissure. Also gliotic localized atrophic changes were observed at the right posterior horn of the lateral ventricle. The patient was referred to the cardiology department for differential diagnosis. Initial physical examination and electrocardiography were evaluated as normal. Transthoracic and then transesophageal echocardiography revealed a hypodense fibrillated mobile $5 \mathrm{~mm}$ mass at the A2 scallop of the anterior mitral valve leaflet and moderate mitral regurgitation (Figures 1-3). Both MRI and transesophageal echocardiography (TEE) findings were consistent with endocarditis, so the patient was admitted to the cardiology department and consulted with rheumatology and infectious diseases. An empiric antibiotic regimen was started after blood culture sampling. Meanwhile her laboratory findings demonstrated all increased positive results of anti-b-2-glycoprotein-1 IgM and IgG, lupus anticoagulant, anti-cardiolipin IgM and IgG and decreased C4 levels. She had no fever and blood cultures were negative, so antibiotherapy was stopped at day 10, with the final diagnosis of primary APS and Libman-Sacks endocarditis. She was discharged with warfarin therapy.

The common cardiac manifestations of APS include valvular involvement such as thickening, vegetations and dysfunction, coronary thrombosis, ventricular hypertrophy and dysfunction, intracardiac thrombi and pulmonary hypertension [2]. The most common echocardiographically

\author{
Corresponding author: \\ Ismail Biyik \\ Department of Cardiology \\ Usak University \\ Education and Research \\ Hospital \\ 64100 Usak, Turkey \\ Fax: +90 5424173209 \\ E-mail: ismailbiyikmd@ \\ yahoo.com
}




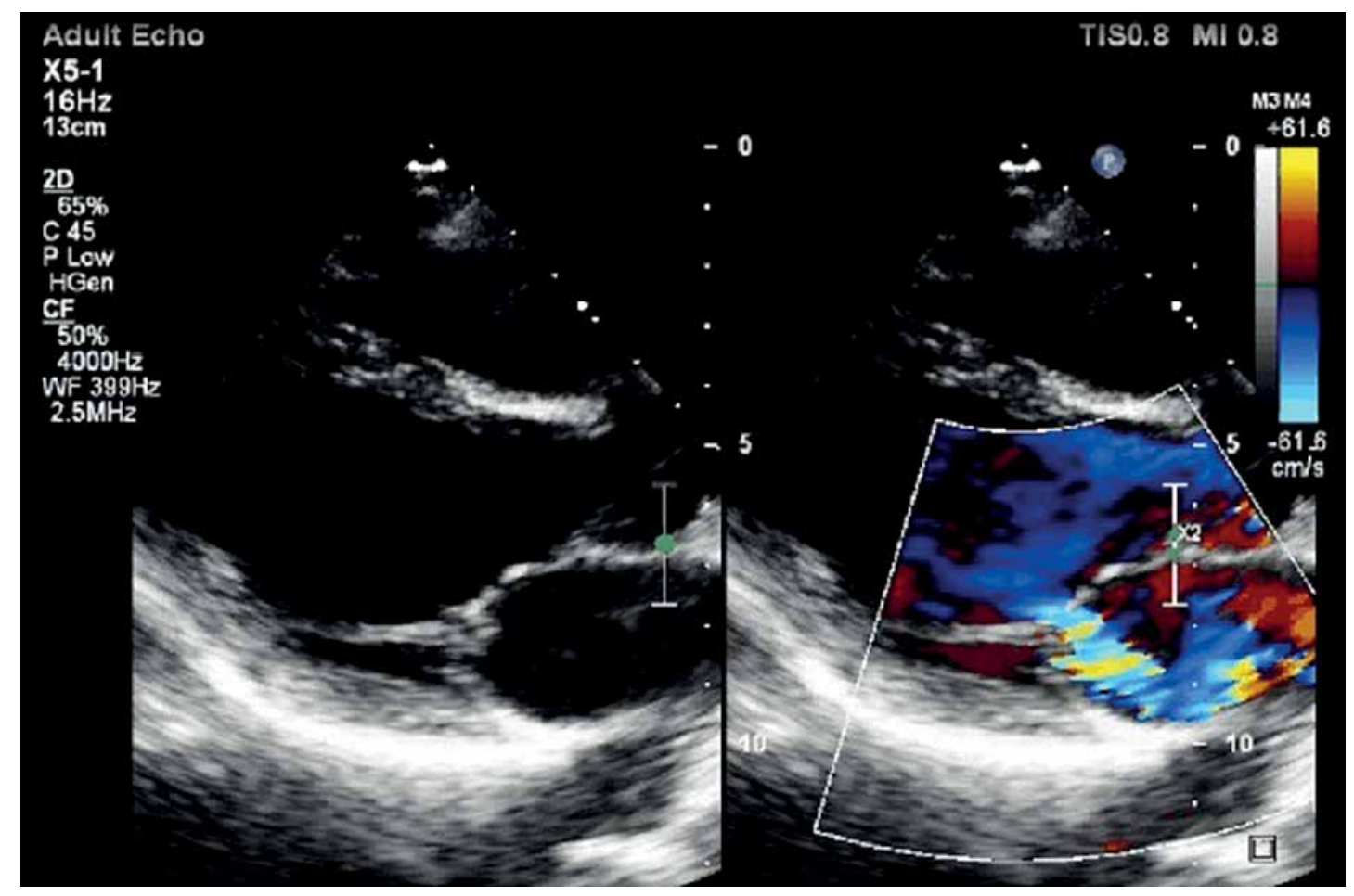

Figure 1. Transthoracic images of vegetations and mitral regurgitation

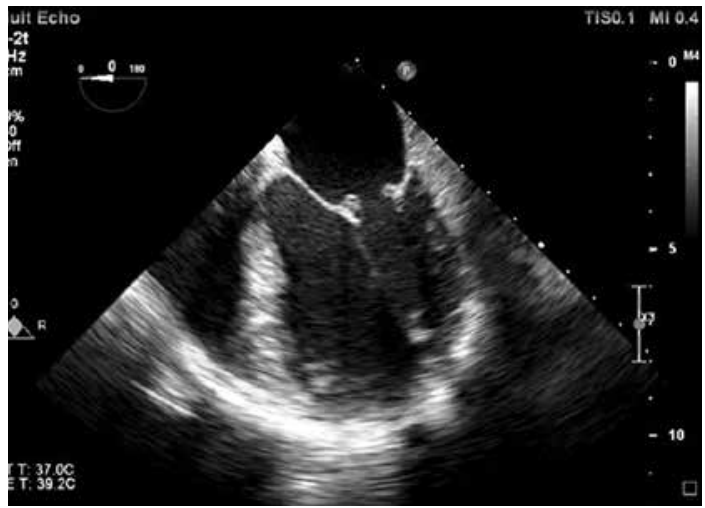

Figure 2. Transesophageal image of vegetations on the coaptation surface of mitral leaflets

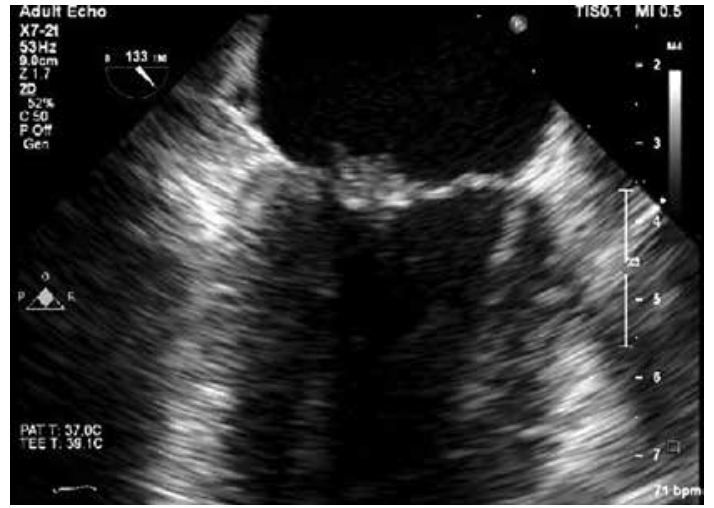

Figure 3. Transesophageal image of kissing vegetations on the edge of mitral leaflets

ic echocardiography is normal, transesophageal echocardiography may be indicated to assess for vegetations due to nonbacterial endocarditis $[4,9]$. The deposition of immunoglobulins on the valve leaflets underlies the pathophysiology of Libman-Sacks endocarditis [10]. aPL not only lead to the formation of verrucous vegetations but also can lead to the formation of valve thrombi and consequently serious complications such as cerebral thromboembolism can occur [6-8]. This was demonstrated in our patient once. This thromboembolic event is one of the most common manifestations in APS patients with Libman-Sacks endocarditis [6-10].

Vegetations may be solitary or multiple and occur in $10 \%$ to $40 \%$ of patients $[3,6]$. They are 
typically irregular in shape and may or may not be mobile [4]. Vegetations on the mitral valve generally form on the atrial surface of leaflets $[3,6]$. Previous reports have also described "kissing lesions" located on the opposing lines of leaflet closure of both the mitral and the aortic valves such as in our case $[3,5,6,8]$. Decreased leaflet mobility and subvalvular thickening are rarely seen [3]. Hemodynamically significant valvular stenosis and regurgitation occur in only $3 \%$ of cases $[3,8$, 9]. There is a paucity of data documenting the echocardiographic response of APS valve disease to antithrombotic therapy. Although anecdotal reports have demonstrated echocardiographic resolution of valvular lesions with treatment, several small series have shown that these may not respond to, or may even progress despite therapy [10]. Surgical intervention is rarely required, which reflects the lack of serious valve deformity characteristic of the disease.

Cardiac involvement occurs frequently and may take a variety of forms. Our case highlights the role of echocardiography and laboratory testing in the detection of APS with primary cardiac involvement.

\section{Conflict of interest}

The authors declare no conflict of interest.

\section{References}

1. Miyakis S, Lockshin MD, Atsumi T, et al. International consensus statement on an update of the classification criteria for definite antiphospholipid syndrome (APS). J Thromb Haemost 2006; 4: 295-306.

2. Tenedios F, Erkan D, Lockshin MD. Cardiac involvement in the antiphospholipid syndrome. Lupus 2005; 14: 691-6.

3. Lan LW, Tsai C, Hou CJ. The valvular involvement of lupus: congestive heart failure can be the presenting feature of systemic lupus erythematosus. Acta Cardiol Sinica 2005; 21: 111-5.

4. Mavrogeni SI, Sfikakis PP, Kitas GD, Kolovou G, Tektonidou MG. Cardiac involvement in antiphospholipid syndrome: the diagnostic role of noninvasive cardiac imaging. Semin Arthritis Rheum 2016; 45: 611-6.

5. Denas G, Jose SP, Bracco A, Zoppellaro G, Pengo V. Antiphospholipid syndrome and the heart: a case series and literature review. Autoimmun Rev 2015; 14: 214-22.

6. Akhlaq A, Ali TA, Fatimi SH. Mitral valve replacement in systemic lupus erythematosus associated Libman-Sacks endocarditis. J Saudi Heart Assoc 2016; 28: 124-6.

7. D'Alton JG, Preston DN, Bormanis J, Green MS, Kraag GR. Multiple transient ischemic attacks, lupus anticoagulant and verrucous endocarditis. Stroke 1985; 16: 512-4.

8. Bourré-Tessier J, Huynh T, Clarke AE, et al. Features associated with cardiac abnormalities in systemic lupus erythematosus. Lupus 2011; 20: 1518-25.

9. Kampolis C, Tektonidou M, Moyssakis I, Tzelepis GE, Moutsopoulos H, Vlachoyiannopoulos PG. Evolution of cardiac dysfunction in patients with antiphospholipid antibodies and/or antiphospholipid syndrome: a 10- year follow-up study. Semin Arthritis Rheum 2014; 43 : 558-65.

10. Tanawutti T, Dia M, Hanif T, Mihailescu M. Double-valve Libman-Sacks endocarditis causing ventricular fibrillation cardiac arrest. Tex Heart Inst J 2011; 38: 295-7. 